\title{
Research and Investigation of Cultural Heritage within the Interdisciplinary CIMA Project
} \author{
and Robert Sablatnig4 \\ ${ }^{1}$ Institute of Science and Technology in Art, Academy of Fine Arts Vienna, Schillerplatz 3, 1010 Vienna, Austria, Europe \\ ${ }^{2}$ Institute of Slavoniic Studies, University of Vienna, Spitalgasse 2, Hof 3, 1090 Vienna, Austria, Europe \\ ${ }^{3}$ Institute of Byzantine and Modern Greek Studies, University of Vienna, Postgasse 7, 1010 Vienna, Austria, Europe \\ ${ }^{4}$ Computer Vision Lab, Vienna University of Technology, Favoritenstrasse 9-11, 1040 Vienna, Austria, Europe
}

Bernadette Fruehmann ${ }^{1 *}$, Federica Cappa ${ }^{1}$, Wilfried Vetter ${ }^{1}$, Manfred Schreiner ${ }^{1}$, Heinz Miklas ${ }^{2}$,Claudia Rapp ${ }^{3}$

Submission: December 15, 2017; Published: April 04, 2018

*Corresponding author: Bernadette Fruehmann, Institute of Science and Technology in Art, Academy of Fine Arts Vienna, Schillerplatz 3, 1010

Vienna, Austria, Europe, Tel: +43 1588 16-8612; Email: b.fruehmann@akbild.ac.at

Keywords: CIMA; palimpsests

Abbreviations: CIMA: Centre of Image and Material Analysis in Cultural Heritage; MSI: Multispectral imaging; LDA: Linear Discriminant Analysis; PCA: Principal Component Analysis; ICA: Independent Component Analysis; XRF: X-ray Fluorescence; rFTIR: Fourier Transform Infrared spectrometry in the reflection mode

\section{Mini-Review}

During the last decades a particular collaboration between representatives of various humanities such as philology, art history and conservation-restoration on the one side and natural scientists on the other has been established, completed by a cooperation with computer vision specialists. Simultaneously, the booming development of analytical methods has offered the possibility to use a great number of new instrumental microanalytical techniques with non-sampling and in-situ applicability for the analysis of written heritage objects $[1,2]$.

In order to give this collaboration a solid structure, the Centre of Image and Material Analysis in Cultural Heritage (CIMA) was established in 2014 with the aid of the Structural Fund for Austrian Higher Education. The main idea behind the foundation of CIMA was to prolong and intensify the cooperation of different institutions by establishing a central laboratory offering its services to universities, libraries, museums, exhibitions, etc. Within this center philologists of the University of Vienna share

their expertise with computer specialists of the TU-Wien and material scientists of the Academy of Fine Arts Vienna. Presently the focus of CIMA is the investigation of (predominantly damaged) medieval manuscripts. Within this framework a series of historical manuscripts of various libraries was examined. The selection comprises badly preserved or rewritten manuscripts (palimpsests) on the one hand, and manuscripts with a remarkable design on the other, deriving from the 9thto the 14th centuries. Mainly Slavic, Greek, and Latin parchment manuscripts from the Austrian National Library were analyzed so far together with codices of various monasteries and libraries in Europe: Kremsmünster Abbey (Austria), Abbey Heiligenkreuz (Austria), Rila Monastery (Bulgaria), National Library Sofia and Plovdiv (Bulgaria), Bibliotheca Comunale Trento (Italy), National and University Library Ljubljana (Slovenia), National Library Budapest (Hungary), National Library Zagreb (Kroatia) and finally also of the St. Catherine Monastery in Sinai (Egypt).

In order to raise the visible contrast of such faded historical documents, multispectral imaging (MSI) is used as a first step of investigation (Figure 1). The manuscripts are examined with a portable MSI acquisition system containing two different cameras:

i. A traditional RGB camera (Red Green Blue - Nikon D4 with a resolution of $4928 \times 3280$ px.), which is used for capturing UV fluorescence and visible light images.

ii. A Phase One IQ260 achromatic multispectral camera with a resolution of 8984 x 6732 pixels. The spectral sensitivity of this second camera reaches from the UV to the near IR-range (350 $\mathrm{nm}$ to $950 \mathrm{~nm}$ ). 


\section{Global Journal of Archaeology \& Anthropology}

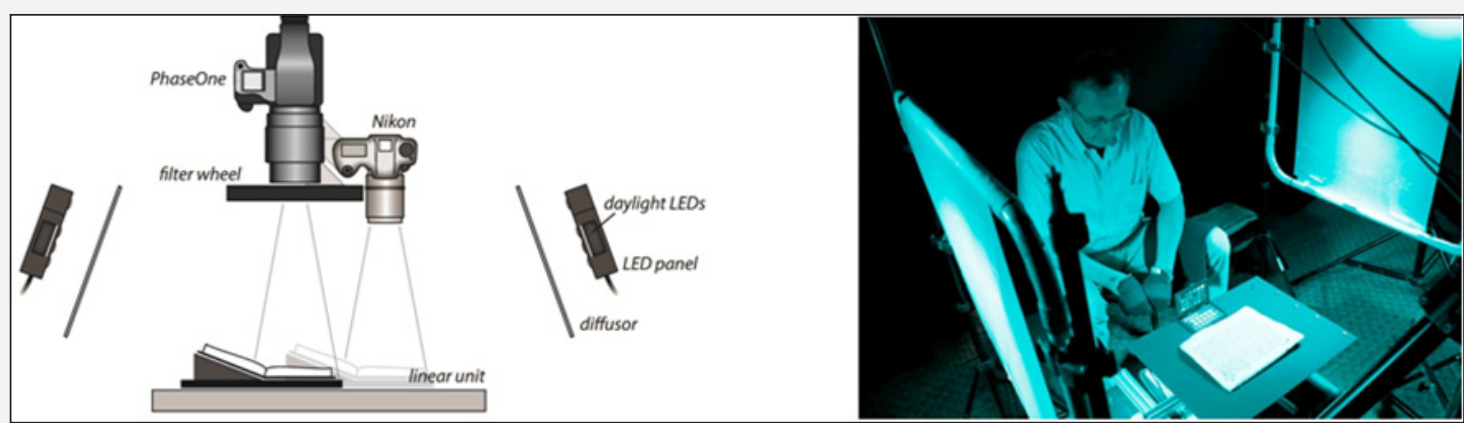

Figure 1: Illustration of the MSI acquisition setup. (a) The objects investigated are placed on a board, which is attached to a linear unit permitting automatic shifting between the two camera positions. The LED system is an Eureka! Light TM system with 11 different wavelengths. For the visible light images white LED panels are attached to the left and right of the Eureka panels. Additionally, two diffusers are positioned between the lighting device and the object in order to uniformly distribute the illumination. (b) Setup during a MSI documentation in the National Library Plovdiv (Bulgaria).

The lighting is provided by two LED panels which enable imaging in 11 narrowband spectral ranges. The imaging in narrow spectral ranges lead to enhancement of the contrast of degraded characters in a manuscript compared to white light illumination (e.g. faded script is usually most visible in UV fluorescence images). In order to further increase the contrast of the degraded characters, several enhancement techniques are applied: Principal Component Analysis (PCA), Independent Component Analysis (ICA) and Linear Discriminant Analysis (LDA) [3].

The material investigation aims to determine the composition of inks and pigments used for writing and illuminating, as well as the characterization of the support material. For this purpose, a non-destructive and non-invasive analysis is required and the analysis can be accomplished under ambient environmental conditions i.e., no alterations can occur on the manuscripts during or after the procedure. The investigations were carried out by applying three complementary non-destructive and noninvasive methods for elemental as well as compound specific material analyses: X-ray Fluorescence (XRF), Fourier Transform Infrared spectrometry in the reflection mode (rFTIR) and Raman spectrometry [4] (Figure 2). Within these investigations mainly iron gall ink was identified in the black/brown inks, whereas well-known pigments like vermilion, red lead, azurite or indigo were detected in miniature paintings. In some cases a mixture of the various pigments with lead white was verified, likely to achieve a brighter tone. Additionally, the identification of iron gall inks is useful not only from a historical perspective but also for conservation and preservation purposes due to its corrosive nature to the support.

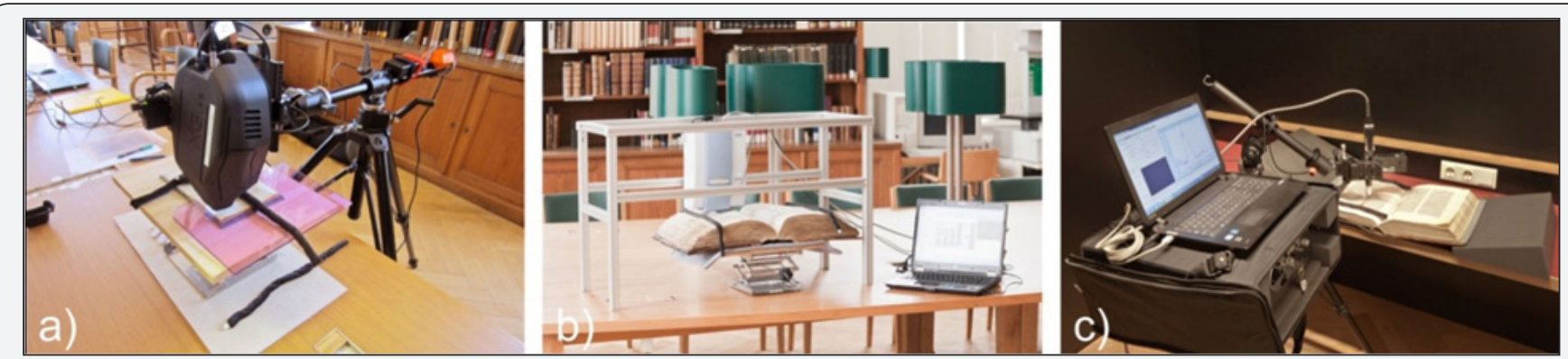

Figure 2 : Investigation of different manuscripts in the Austrian National Library (ÖNB), Vienna.

a) X-ray analysis with the ELIO instrument (XGLab, Italy); b) rFTIR measurements with the ALPHA spectrometer (Bruker Optics, Germany) and c) Raman studies performed with the Pro-Raman-L-Dual-G system (Enwave, USA).

In the course of the project, a common database will be implemented which contains the information gained from the imaging, image enhancement as well as chemical and philological investigations. The final objective of the research is to compare the data gained to reveal correlations within data stemming from multiple modalities and, in general, to make new scholarly and scientific findings.

\section{References}

1. Janssens K, Van Grieken R (2004) Non Destructive Microanalysis of Cultural Heritage Materials, Vol. XLII, in Comprehensive Analytical Chemistry edited by D. Barceló, Wilson \& Wilson’s, Elsevier Amsterdam.

2. Schreiner M, Wiesinger R, Vetter $W$ (2017) Identification and Preservation of Cultural Heritage, Wiley-VCH Verlag GmbH \& Co. KGaA 
3. Camba M, Gau F, Hollaus S, Fiel R Sablatnig (2014) Multispectra Imaging, Image Enhancement, and Automated Writer Identification in Historical Manuscripts Cultures. In: Brockmann CH, Friedrich M, et al., Volume 7, Manuscript Cultures.

This work is licensed under Creative Commons Attribution 4.0 License

DOI: 10.19080/GJAA.2018.03.555614
4. Fruehmann F, Cappa W, Vetter M, Schreiner (2017) Three complementary non-invasive methods applied to historical manuscripts, , in Manuscript Cultures. In: Brockmann CH, Friedrich M, et al. (Eds.), Hamburg.

\section{Your next submission with Juniper Publishers will reach you the below assets}

- Quality Editorial service

- Swift Peer Review

- Reprints availability

- E-prints Service

- Manuscript Podcast for convenient understanding

- Global attainment for your research

- Manuscript accessibility in different formats

( Pdf, E-pub, Full Text, Audio)

- Unceasing customer service

Track the below URL for one-step submission https://juniperpublishers.com/online-submission.php 